\title{
Aperiodic non-isomorphic lattices with equivalent percolation and random-cluster models.
}

\author{
Klas Markström* and John C. Wierman ${ }^{\dagger}$ \\ Submitted: May 6, 2009; Accepted: Mar , 2010; Published: Mar 29, 2010 \\ Mathematics Subject Classification: 60K35, 05C80,05C65
}

\begin{abstract}
We explicitly construct an uncountable class of infinite aperiodic plane graphs which have equal, and explicitly computable, bond percolation thresholds. Furthermore for both bond percolation and the random-cluster model all large scale properties, such as the values of the percolation threshold and the critical exponents, of the graphs are equal. This equivalence holds for all values of $p$ and all $q \in[0, \infty]$ for the random-cluster model.

The graphs are constructed by placing a copy of a rotor gadget graph or its reflection in each hyperedge of a connected self-dual 3-uniform plane hypergraph lattice. The exact bond percolation threshold may be explicitly determined as the root of a polynomial by using a generalised star-triangle transformation. Related randomly oriented models share the same bond percolation threshold value.
\end{abstract}

\section{Introduction and Summary}

Although it has been over fifty years since the origins of percolation theory, until recently the percolation threshold had been exactly determined for only a small collection of lattice graphs. Periodicity has been a crucial assumption for rigorous proofs of all these exact percolation threshold values. Research of Scullard [10], Ziff [20], Scullard and Ziff [11], and Ziff and Scullard [21] used a generalised star-triangle transformation and a concept of triangle-duality to predict exact solutions for a collection of periodic lattice graphs in two dimensions. Wierman and Ziff [19] organised this approach to describe a construction of an infinite class of planar graphs for which the bond percolation threshold can be mathematically rigorously determined. The graphs are constructed by placing isomorphic copies of a finite connected planar graph with three terminals, called a generator, in a connected self-dual planar periodic 3-uniform hypergraph structure. A consequence of

\footnotetext{
*Department of Mathematics and Mathematical Statistics, Umeå University, Sweden

${ }^{\dagger}$ Department of Applied Mathematics and Statistics, Johns Hopkins University, USA
} 
the construction is that for each of an infinite set of real numbers $a \in(0,1)$ there are a countable infinite family of periodic lattices which have bond percolation threshold equal to $a$. Sedlock and Wierman [12] generalised an argument of Wierman [18] using the substitution method (See [2, Ch.6]) to show that the critical exponents are equal for any dual pair of lattices constructed by the method. Placing the same generator and its dual generator in the countably infinite collection of self-dual hypergraphs identified by Wierman and Ziff [19] creates a countably infinite set of lattices which have the same critical exponents. This observation provided supporting evidence for the universality hypothesis that the critical exponents agree for all two-dimensional percolation models.

In this paper we extend these results in several ways. First we construct an uncountably large family of infinite planar graphs, most of which have only the trivial automorphism, with identical bond percolation thresholds. However unlike the previously mentioned construction the percolative behaviour is identical not only at the threshold $p_{c}$ but at all values of the edge probability $p$. Furthermore this is also extended to the general random-cluster model for all values of the parameter $q$. We recall that $q=1$ corresponds to ordinary percolation and $q=2$ to the Ising model. As before this means that the critical exponents for the general random-cluster model are also equal for all graphs in the family.

The graphs are constructed using a generator which exhibits three-fold rotational symmetry, but have no other automorphisms. In particular, it is not symmetric respect to reflection in an axis. Such a graph, called a rotor gadget, arises in the study of the Tutte polynomial [17] and isomagnetic graphs [1]. The graphs are constructed by deterministically placing either the rotor gadget or its reflection in each of the hyperedges of the hypergraph structure. Most of the resulting graphs do not have the periodicity required by the classical methods to rigorously determine exact percolation thresholds. The results are instead obtained by applying classical methods to a periodic lattice, then modifying the lattice without changing the connection probabilities between terminals of a generator, using the the properties of the rotor gadget. The exact bond percolation threshold can be determined using the generalised star-triangle transformation. Definitions of elements of the construction and proof method are provided in section 2. Construction of the aperiodic graphs and the proof that their bond percolation thresholds are equal are given in section 3.1 .

Since all graphs in the class have the same percolation threshold, in section 3.2 we consider randomly-oriented models in which the placement of the rotor gadget and its reflection in hyperedges is made according to a probability measure, showing that all such models share the same percolation threshold as the deterministic models.

In section 3.3, the results of Sedlock and Wierman [12] are applied to show that the set of critical exponents of bond percolation on these graphs and their dual graphs are identical, under the assumption that the limits defining the critical exponents exist. This result extends to the randomly-oriented models also.

Finally, in section 4, the random-cluster model is considered on these graphs, showing that common critical points and critical exponents are shared by all these graphs, due to the identity of the connection probabilities and cluster numbers in the rotor graph and its 
reflection. Although it rarely is the case for the random-cluster model, the result holds for both the $q<1$ and $q \geqslant 1$ cases.

\section{Definitions and Preliminaries}

\subsection{Bond Percolation Threshold}

Let $G$ be a connected graph, and $p \in(0,1)$ be a real-valued parameter. Let $E(G)$ denote the edge set of $G$. In the bond percolation model on $G$, each $e \in E(G)$ is assigned a state, open or closed. Representing the states open and closed as 0 and 1 , respectively, a realisation of the model may be identified with an element $\omega \in \Omega=\{0,1\}^{E(G)}=2^{E(G)}$, called a configuration. The states of the edges are mutually independent, with each edge open with probability $p$. The corresponding product probability measure on $2^{E(G)}$ is denoted by $P_{p}$.

The number of open edges in $\omega$ is denoted $|\omega|$. An open cluster is a maximal connected subgraph of $G$ which has all edges open. The size of the cluster $C$, denoted $|C|$, is the number of edges in $C$. The number of open clusters in $\omega$ is denoted $k(\omega)$.

Now assume that $G$ is an infinite locally-finite graph. Let $E_{\infty}$ denote the event that there is an infinite open cluster in $G$. A standard coupling argument shows that $P_{p}\left[E_{\infty}\right]$ is nondecreasing in $p$, and Kolmogorov's zero-one law shows that its value is either 0 or 1 for any $p$. Thus, there exists a bond percolation threshold $p_{c}(G)$ such that $P_{p}\left[E_{\infty}\right]=0$ for $p<p_{c}(G)$ and $P_{p}\left[E_{\infty}\right]=1$ for $p>p_{c}(G)$.

\subsection{Plane Lattice}

A plane graph is a graph that is drawn in the plane with non-crossing edges. Typically, the edges are drawn as straight line segments, but we may assume that they are drawn as piecewise linear curves. A plane graph is periodic if there are two basis vectors $u, v \in \mathbf{R}^{2}$ such that the translation of $\mathbf{R}^{2}$ by any linear combination of the $u$ and $v$ with integer coefficients induces an isomorphism of $G$ as a plane graph. By a plane lattice we mean a periodic plane graph $G$ which is connected, infinite, and locally finite, and the vertex set is a subset of $\mathbf{R}^{2}$ which has no accumulation points.

Given a plane graph $G$, its dual graph, denoted $G^{*}$, is the graph whose vertices are in one-to-one correspondence with the faces of $G$, and whose edges are in one-to-one correspondence with the edges of $G$ : Each vertex of $G^{*}$ lies in the corresponding face of $G$. For each edge $e \in E(G)$, its dual edge $e^{*}$ joins the vertices of $G^{*}$ corresponding to the faces of $G$ in which it lies. Note that if $G$ is a plane lattice, then an appropriate drawing of $G^{*}$ is also.

\subsection{Generator and Dual Generator}

In this article, a generator is a finite plane graph $G$ which is connected and has three vertices, referred to as boundary vertices, attachment points, or terminals, among the 
vertices on the boundary of the unbounded face. A generator $G$ will be drawn in the plane with its boundary vertices, labelled counterclockwise as $A, B$, and $C$, at the vertices of the triangle, and all vertices and edges of $G$ contained in the triangle and its interior.

Given a generator $G$, its dual generator $G^{*}$ is a graph with a vertex located in each bounded face of $G$ and three vertices, labelled $A^{\prime}, B^{\prime}$, and $C^{\prime}$, in the unbounded face, and edges of $G^{*}$ in one-to-one correspondence with the edges of $G$. For each edge $e \in E(G)$, its dual edge $e^{*}$ is incident to the vertices of $G$ corresponding to the faces in which it lies, with the following provision for edges $e^{*}$ with an endvertex in the unbounded face of $G$ : Let $\pi_{A B}$ denote the path of edges in $G$ which is on the boundary of the unbounded face of $G$ and does not pass through $C$, and define $\pi_{B C}$ and $\pi_{A C}$ similarly. If $e \in \pi_{A B}$, connect its dual edge $e^{*}$ to $C^{\prime}$. If $e \in \pi_{B C}$, connect $e^{*}$ to $A^{\prime}$. If $e \in \pi_{A C}$, connect $e^{*}$ to $B^{\prime}$. Clearly, $G^{*}$ is not the dual graph of $G$, but is the appropriate graph to use in the construction of dual lattices constructed from $G$.

\subsection{Partition Probabilities}

To describe the probabilities of open connections within a 3-terminal generator $G$, we consider the probabilities of the five possible partitions of the terminals into distinct open clusters. A partition will be denoted by a string of vertices and vertical bars, in which vertices separated by a bar are in different open clusters. The five possible partitions are $A B C, A B|C, A C| B, A \mid B C$, and $A|B| C$. In a percolation model with parameter $p$, the probability of each partition will be a polynomial function of $p$. As $p$ increases from 0 to $1, P_{p}[A B C]$ increases from 0 to 1 , while $P_{p}[A|B| C]$ decreases from 1 to 0 . Given a generator $G$, let $p_{0}(G)$ denote the value of $p$ such that these probabilities are equal. As a consequence of a generalised star-triangle transformation described in Wierman and Ziff [19], $p_{0}(G)$ determines the exact bond percolation threshold of the lattice constructed from a generator $G$ as in the following paragraphs.

\subsection{Plane Hypergraph Lattice}

A hypergraph $\mathcal{H}$ consists of a vertex set $V(\mathcal{H})$ and a set $E(\mathcal{H})$ of subsets of $V(\mathcal{H})$ called hyperedges. A $k$-hyperedge is a hyperedge that contains exactly $k$ vertices. A $k$-uniform hypergraph is one in which every hyperedge is a $k$-hyperedge. In this article, we will restrict consideration to 3-uniform hypergraphs.

A plane hypergraph is a hypergraph that is drawn in the plane so that each hypergraph vertex is represented by a point in the plane, each hyperedge is represented by a region bounded by a polygon with its hyperedge vertices located at vertices of the polygon, and the polygons intersect only at hyperedge vertices. A hypergraph $\mathcal{H}$ is connected if for any $x, y \in V(\mathcal{H})$ there is a sequence $x=w_{0}, w_{1}, w_{2}, \ldots, w_{n}=y \in \mathcal{H}$ such that $w_{i-1}$ and $w_{i}$ are in a common hyperedge for each $i=1, \ldots, n-1$, . A hypergraph is locally finite if each of its vertices is in only a finite set of hyperedges. As for plane graphs, a plane hypergraph is periodic if there are two basis vectors $u, v \in \mathbf{R}^{2}$ such that the translation of $\mathbf{R}^{2}$ by any integer linear combination of $u$ and $v$ induces an isomorphism of $\mathcal{H}$ as a plane hypergraph. 
A plane hypergraph lattice is a periodic plane hypergraph which is connected, infinite, and locally finite, and the vertex set is a subset of $\mathbf{R}^{2}$ which has no accumulation points.

\subsection{Dual and Self-Dual Hypergraphs}

Given a plane hypergraph lattice $\mathcal{H}$ embeded in the plane, the complement to the set of points inside the edges of $\mathcal{H}$ in the plane is a union of polygonal faces. Construct the dual plane hypergraph lattice $\mathcal{H}^{*}$ as follows: [The reader should note that this concept of plane duality differs from the standard hypergraph duality concept.] The vertices of $\mathcal{H}^{*}$ are in one-to-one correspondence with the faces of $\mathcal{H}$, represented by a point in the face, see Figure 1. The hyperedges of $\mathcal{H}^{*}$ are in one-to-one correspondence with the hyperedges of $\mathcal{H}$. For each hyperedge $H \in \mathcal{H}$, the dual hyperedge $H^{*}$ is represented by a polygonal region with the hypergraph vertices of $H^{*}$ in the faces of $\mathcal{H}$ neighbouring $H$, and which does not intersect the polygonal region of any hyperedge of $\mathcal{H}$ other than $H$. Note that since $\mathcal{H}$ is a plane hypergraph lattice, there is an appropriate drawing of $\mathcal{H}^{*}$ in which the hyperedges of $\mathcal{H}^{*}$ do not intersect except at vertices of $\mathcal{H}^{*}$, and which is periodic. Thus, there is a drawing such that $\mathcal{H}^{*}$ is also a plane hypergraph lattice.

A plane hypergraph lattice $\mathcal{H}$ is self-dual if $\mathcal{H}^{*}$ and $\mathcal{H}$ are isomorphic as graphs. Self-dual plane hypergraph lattices play a major role in the determination of exact bond percolation thresholds for plane periodic lattices. Wierman and Ziff [19] illustrate several examples of self-dual plane hypergraph lattices, and prove that there are infinitely many.

\subsection{Lattice Construction}

Given a self-dual plane hypergraph lattice $\mathcal{H}$ and a generator $G$, we now construct a pair of dual plane lattices as follows: Embed the generator $G$ in each hyperedge of $\mathcal{H}$ with the terminals of $G$ located at the vertices of the hyperedge, with each copy of $G$ contained in the region representing the corresponding hyperedge, with the set of copies having a specified orientation $\mathcal{O}$ so that the resulting plane graph is periodic. Denote the resulting plane lattice by $L(G, \mathcal{H}, \mathcal{O})$. Construct a drawing of the dual plane hypergraph lattice $\mathcal{H}^{*}$, and embed copies of the dual generator $G^{*}$ in the regions corresponding to the hyperedges of $\mathcal{H}^{*}$ according to the orientation $\mathcal{O}^{*}$ determined by the convention specified above in the definition of dual generator, to obtain a plane lattice $L\left(G^{*}, \mathcal{H}^{*}, \mathcal{O}^{*}\right)$. The infinite graphs $L(G, \mathcal{H}, \mathcal{O})$ and $L\left(G^{*}, \mathcal{H}^{*}, \mathcal{O}^{*}\right)$ are a dual pair of plane lattices, to which we may apply standard results from bond percolation theory. For a given lattice $L$ we call the set of vertices which correspond to the terminal vertices, or equivalently the vertices of the hypergraph, the hubset of the lattice and denote it by $H u b(L)$.

\subsection{Symmetry Considerations}

A plane lattice $L$ or plane hypergraph lattice $\mathcal{H}$ has $k$-fold symmetry, for $k \geqslant 2$, if it is invariant under rotation about the origin through an angle of $2 \pi / k$. A plane lattice $L$ 


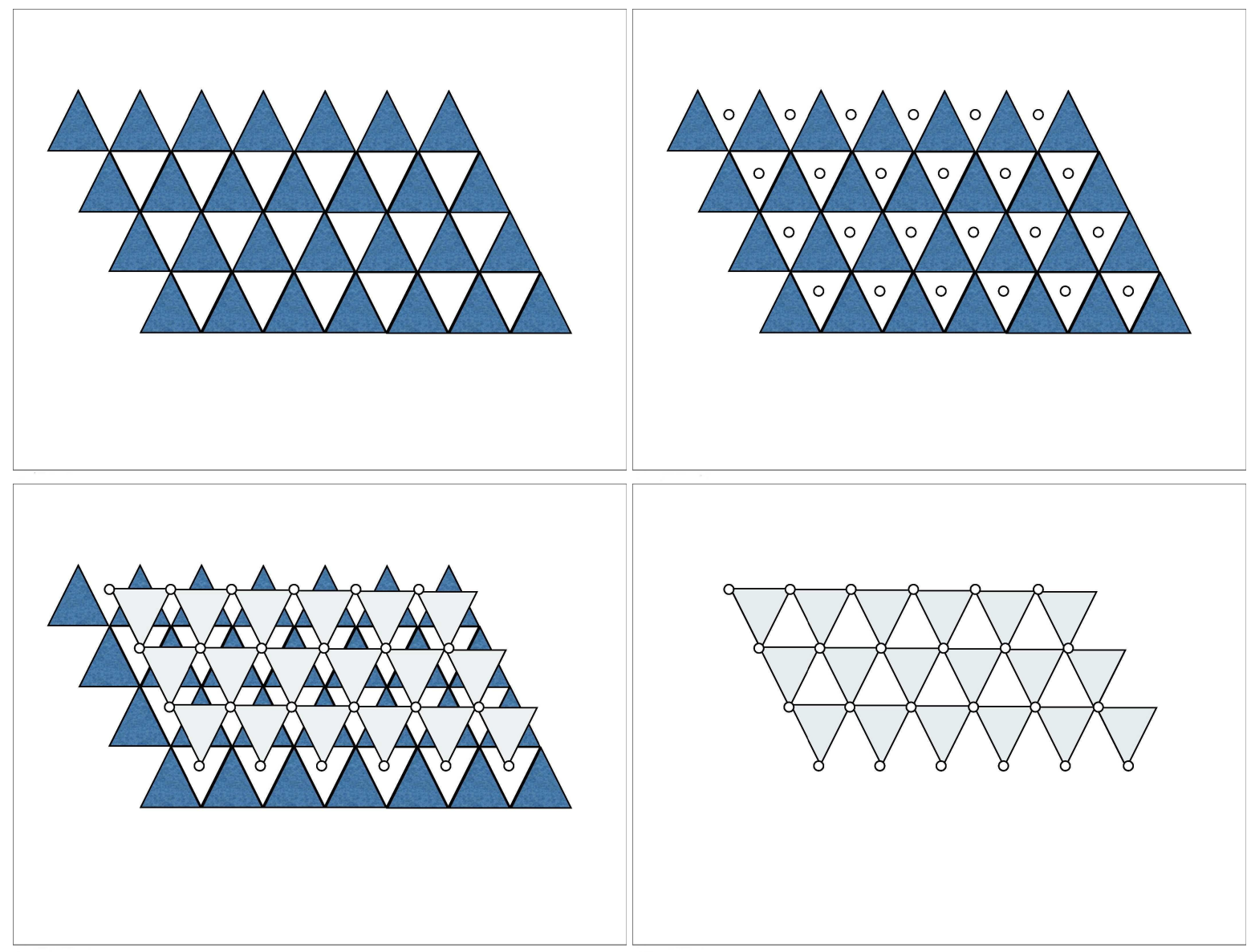

Figure 1: Part of a plane hypergraphs lattice, with edges coloured (Top Left), The lattice and the vertices of the dual (Top Right), The lattice and with the dual superimposed (Bottom Left), The dual (Bottom Right)

or plane hypergraph lattice $\mathcal{H}$ has reflection symmetry if it is invariant under reflection through a line.

Classical percolation methods which are used to obtain exact percolation threshold values require reflection symmetry [9] or $k$-fold symmetry [3]. Note that all the self-dual plane hypergraph lattices described in [19] have reflection symmetry or a $k$-fold symmetry. A result of Sheffield [14] applies to all plane lattices with no such symmetry requirements [3]. We state our results without requiring symmetry, since it is plausible that there exist self-dual planar hypergraphs which do not exhibit any symmetry. However the examples we have explicitly considered are symmetric enough to be dealt with by less intricate methods than those used by Sheffield.

\subsection{Rotor Gadget}

A rotor gadget is a finite plane graph with three terminal vertices such that the graph has rotational symmetry, and the three terminals belong to the same orbit under the rotation, 

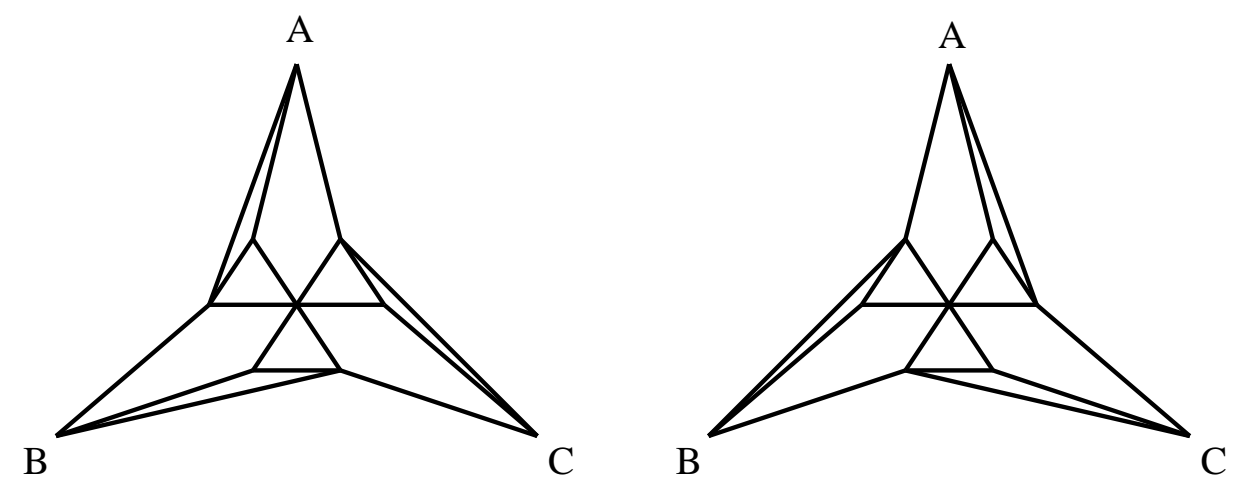

Figure 2: A rotor gadget and its reflection.

but has no other automorphisms. In Figure 2 we show an example of a rotor gadget.

Since we consider only rotor gadgets as generators, in this article we suppress mention of orientation in the notation for lattices. By using suitable subdivision and addition of edges it is easy to see that the set of non-isomorphic rotor gadgets is countably infinite.

Rotor gadgets were introduced by Tutte in his study of the Tutte polynomial and chromatic polynomials. (See [17] for an enjoyable introduction and historical survey.) Later [1] they were also used to show that almost all planar triangulations are not determined by their bivariate Ising polynomial.

\section{$3 \quad$ Results and Proofs for Percolation}

\subsection{Aperiodic Graphs}

Our aim is to prove that an uncountable family of aperiodic planar graphs have equal bond percolation thresholds. We may construct such a family from a rotor gadget $R$ and a self-dual plane hypergraph lattice $\mathcal{H}$. Each $\rho \in\left\{R, R^{\prime}\right\}^{E(\mathcal{H})} \equiv 2^{E(\mathcal{H})}$, corresponds to an assignment of the rotor graph $R$ or its reflection $R^{\prime}$ to each hyperedge of $\mathcal{H}$. Let $L(R, \mathcal{H}, \rho)$ denote the graph constructed by embedding the assigned generator in each hyperedge of $\mathcal{H}$.

Lemma 3.1. For each rotor gadget $R$ and self-dual plane hypergraph lattice $\mathcal{H}$, the set $\left\{L(R, \mathcal{H}, \rho): \rho \in 2^{E(\mathcal{H})}\right\}$ contains uncountably many non-isomorphic graphs.

Proof. The set $E(\mathcal{H})$ of hyperedges of $\mathcal{H}$ is infinite, so $2^{E(\mathcal{H})}$ is uncountable. However, many of these lattices may be isomorphic via translation, rotation, or reflection. Since the automorphism group of translations, rotations, and reflections is countable, each isomorphism class is countable. Thus, the set of isomorphism classes is uncountable.

Since many of the graphs $L(R, \mathcal{H}, \rho)$ are aperiodic, standard percolation results do not directly apply. However, we may apply the classical methods to the plane lattice $L(R, \mathcal{H})=L\left(R, \mathcal{H}, R^{E(\mathcal{H})}\right)$ which has the rotor gadget $R$ embedded in all hyperedges of $\mathcal{H}$. 
Proposition 3.2. For any rotor gadget $R$ and self-dual plane hypergraph lattice $\mathcal{H}$,

$$
p_{c}(L(R, \mathcal{H}))+p_{c}\left(L\left(R^{*}, \mathcal{H}\right)\right)=1
$$

and

$$
p_{c}(L(R, \mathcal{H}))=p_{0}(R) .
$$

Proof. Note that $L(R, \mathcal{H})$ and $L\left(R^{*}, \mathcal{H}\right)$ are both periodic, and are dual plane lattices to which the results of Kesten [9], Bollobás and Riordan [3], or Sheffield [10] apply to obtain the first equation. In the approach described by Wierman and Ziff [19], by the generalised star-triangle transformation, the bond percolation threshold for $L(R, \mathcal{H})$ is given by $p_{0}(R)$.

The percolation threshold result may now be transferred to the aperiodic graphs $L(R, \mathcal{H}, \rho)$.

Theorem 3.3. For any rotor gadget $R$ and self-dual plane hypergraph lattice $\mathcal{H}$,

$$
p_{c}(L(R, \mathcal{H}, \rho))=p_{0}(R)
$$

for all $\rho \in 2^{E(\mathcal{H})}$.

Proof. Note that, since $R$ and $R^{\prime}$ are isomorphic as graphs and the partition probabilities do not depend on the embedding, the partition probabilities for $R$ and $R^{\prime}$ are identical for all $p \in(0,1)$. Thus, replacement of $R$ by $R^{\prime}$ in any hyperedge of $L(R, \mathcal{H})$ does not change the connection probabilities between any vertices of the hypergraph. Hence, for any $\rho \in 2^{E(\mathcal{H})}$, the percolation threshold satisfies

$$
p_{c}(L(R, \mathcal{H}, \rho))=p_{c}(L(R, \mathcal{H}))=p_{0}(R),
$$

with the last equality by Proposition 3.2.

Example 3.4. For the rotor gadget in Figure 2 we can compute the partition probabilities by classifying all subsets of the edge set of the rotor gadget. We find that

$$
\begin{aligned}
& P_{p}[A B C]=3 p^{4}+15 p^{5}+73 p^{6}-111 p^{7}-627 p^{8}+902 p^{9}+1872 p^{10}-4242 p^{11} \\
& \quad-1158 p^{12}+11868 p^{13}-16746 p^{14}+12260 p^{15}-5208 p^{16}+1224 p^{17}-124 p^{18}
\end{aligned}
$$

If we solve the equation $P_{p}[A B C]=P_{p}[A|B| C]$ we find that it has a single real root in the interval $(0,1)$ giving us $p_{c}=p_{0}(R) \approx 0.432785$.

Wierman and Ziff [19] showed that there are countably infinitely many different plane graphs $L$ which have a common value for $p_{c}(L)$. An immediate consequence of Proposition 3.1 and Theorem 3.3 is the following stronger result:

Corollary 3.5. For a rotor gadget $R$ and a self-dual plane hypergraph lattice $\mathcal{H}$, there are uncountably many non-isomorphic plane graphs $L(R, \mathcal{H}, \rho)$ with bond percolation threshold equal to $p_{0}(R)$. 


\subsection{Randomly Assigned Generators}

Rather than constructing a graph deterministically, as above, consider randomly placing the rotor gadget as $R$ or its reflection $R^{\prime}$ in each hyperedge in the construction. We may consider any probability measure $Q$ on $2^{E(\mathcal{H})}$, and let $\mathcal{P}$ denote the set of such probability measures. Let $L(R, \mathcal{H}, Q)$ denote the model in which the configuration of generators is distributed according to $Q$. For example, $Q$ may assign $R$ or $R^{\prime}$ with probability $p$ and $1-p$, respectively, to each hyperedge of $\mathcal{H}$ independently. One may also use a dependent measure $Q$, such as that of the spin value in an Ising model.

Corollary 3.6. For any probability measure $Q \in \mathcal{P}$,

$$
p_{c}(L(R, \mathcal{H}, Q))=p_{c}(R)
$$

That is, the percolation threshold is equal for all realisations of the random variable $L(R, \mathcal{H}, Q)$

Proof. Since every assignment of the generators $R$ and $R^{\prime}$ has the same connection probabilities between hyperedge vertices as in $L(R, \mathcal{H})$ for all $p$, the connection probabilities in the random model with probability measure $Q$ are also the same. Thus, the bond percolation thresholds are equal.

A random triangulation model with some similarities was treated by Bollobás and Riordan [3], in which a site percolation model on the square lattice with randomly oriented diagonals was shown to almost always have percolation threshold equal to $1 / 2$. Their model differs in several respects: First, the graphs they considered consisted of a fixed subgraph with additional randomly oriented edges, while our graphs need not have any fixed subgraphs. Secondly, their conclusion holds for almost every, but not for every, realisation of the randomly oriented graph model. Finally their proof requires that a suitable modification of the model is positively correlated, while, as we will see in Section 4, our construction can also be applied to situations with purely negative correlations.

\subsection{Critical Exponents}

Many functions of interest in percolation theory are believed, but not proved, to behave near criticality as powers of $\left|p-p_{c}\right|$ as $p$ approaches $p_{c}$, with the powers called critical exponents. The expression $A(p) \approx\left|p-p_{c}\right|^{\zeta}$ denotes that

$$
\lim _{p \rightarrow p_{c}} \frac{\log A(p)}{\log \left|p-p_{c}\right|}=\zeta .
$$

The most common power laws considered are for the percolation probability function $\theta(p)=P_{p}\left[\left|C_{v}\right|=+\infty\right] \approx\left(p-p_{c}\right)^{\beta}$, for $p>p_{c}$, for some $0<\beta<1$, and the mean finite cluster size $\chi_{f}(p)=E_{p}\left[\left|C_{v}\right| ;\left|C_{v}\right|<\infty\right] \approx\left|p-p_{c}\right|^{-\gamma}$ for some $\gamma>0$. Additional critical exponents considered in the literature are $\eta$ associated with the two-point connectivity at criticality, $\nu$ for the correlation length, $\delta$ for the cluster volume at criticality, $\alpha$ for 
the number of clusters per vertex, and $\Delta$ for the cluster moments. (See [7, Ch.9].) Note, however, that it is not known that the defining limits exist for any bond percolation model in two-dimensions.

The values for these critical exponents are believed to be $\alpha=-\frac{2}{3}, \beta=5 / 36, \gamma=43 / 18$, $\delta=91 / 5$, and $\nu=4 / 3$ for any two-dimensional lattice. Kesten [9] proved that for a broad class of two-dimensional periodic lattices including the plane lattices considered in this article, (assuming the limits defining the exponents $\delta$ and $\nu$ exist)

$$
\beta=\frac{2 \nu}{\delta+1}, \gamma=2 \nu \frac{\delta-1}{\delta+1}, \eta=\frac{4}{\delta+1}, \text { and } \Delta=2 \nu \frac{\delta}{\delta+1} .
$$

A remarkable result by Smirnov and Werner [15] combines Kesten's scaling relations, knowledge of critical exponents associated with the stochastic Loewner evolution process, and Smirnov's proof of conformal invariance to determine the existence and values of critical exponents for site percolation model on the triangular lattice. However, our results do not establish any numerical values for critical exponents.

Theorem 3.7. For a rotor gadget $R$ and a self-dual plane hypergraph lattice $\mathcal{H}$, assuming that the limits defining the critical exponents $\beta, \gamma, \nu, \delta, \eta$, and $\Delta$, exist for the graphs, the values of this set of critical exponents are equal for the bond percolation models $\left\{L(R, \mathcal{H}, \rho): \rho \in 2^{E(\mathcal{H})}\right\},\left\{L\left(R^{*}, \mathcal{H}, \rho\right): \rho \in 2^{E(\mathcal{H})}\right\},\{L(R, \mathcal{H}, Q): Q \in \mathcal{P}\}$, and $\left\{L\left(R^{*}, \mathcal{H}, Q\right): Q \in \mathcal{P}\right\}$.

Proof. Let us first consider the case in which the root vertex $v$ belongs to $H u b(L)$. Due to the symmetry of the generator $R$, the size, radius and other properties of the clusters appearing the definitions of the critical exponents, do not depend on the orientations $\rho$. Additionally since the symmetry group of the planar hypergraph $\mathcal{H}$ acts transitively on the hub vertices the cluster properties will be independent of the choice of $v \in H u b(L)$.

Next we consider a root vertex $v \notin H u b(L)$ which belongs to some copy $R_{1}$ of the generator $R$. Since $R$ is finite there is a path of some length $l_{v}$ from $v$ to some vertex $u \in H u b(l)$. Hence each of the quantities in the definitions of the critical exponents differs by at most a factor of $p^{l_{v}}$, in the case of probabilities, or an additive term of size at most $l_{v}$, in the case of cluster sizes, between $v$ and a vertex $u \in H u b(L)$. However neither bounded additive terms nor constant factors will affect the value of the critical exponent, due to the unbounded denominator in 3 . Hence the expressions defining the critical exponents will give the same values for all vertices.

As noted above, the partition probability functions are identical for all models in $\left\{L(R, \mathcal{H}, \rho): \rho \in 2^{E(\mathcal{H})}\right\}$ and $\{L(R, \mathcal{H}, Q): Q \in \mathcal{P}\}$, so connections between the vertices of the hypergraph have equal probabilities, and thus equal critical exponents, in all these models.

This is also true for $\left\{L\left(R^{*}, \mathcal{H}, \rho\right): \rho \in 2^{E(\mathcal{H})}\right\}$ and $\left\{L\left(R^{*}, \mathcal{H}, Q\right): Q \in \mathcal{P}\right\}$. Sedlock and Wierman [12] show that $L(G, \mathcal{H})$ and $L\left(G^{*}, \mathcal{H}\right)$ have the same set of critical exponent values for any generator $G$, which provides the result.

Note that if the limits defining the critical exponents do not exist, the method of Sedlock and Wierman shows that the lim infs of the quantities are equal and the lim sups of the quantities are equal for these sets of graphs. 


\section{The Random-Cluster Model}

The random-cluster model is a generalisation of percolation where the probability of an edge configuration does not only depend on the number of open edges but also on the number of its components. The model was introduced by Fortuin and Kasteleyn [4] in 1972, but the partition function of the model was introduced by Tutte as an object in algebraic graph theory already in 1947 [16].

For a finite graph $G$ with $m$ edges the probability of a configuration $\omega \in 2^{E(G)}$ with $|\omega|$ open edges and $k(\omega)$ components is

$$
\phi_{p, q}(G, \omega)=\frac{1}{Z_{p, q}} p^{|\omega|}(1-p)^{m-|\omega|} q^{k(\omega)}
$$

where $Z_{p, q}$ is a normalising constant.

For an infinite graph there are several different ways to define the random-cluster measure on $2^{E(G)}$ (See [8] for a more detailed discussion.), but one possibility is to require the correct marginal distribution on finite subgraphs.

Given finite $\Lambda \subset V(G)$ and $\zeta \in 2^{E(G)}$ let $\Omega_{\Lambda}^{\zeta}$ denote the finite subset of $2^{E(G)}$ such $\omega(e)=\zeta(e)$ for $e \in E(G) \backslash E(\Lambda)$. Let $k(\omega, \Lambda)$ denote the number of components of $\omega$ that intersect $\Lambda$.

We now define the random-cluster measure with boundary condition $\zeta$ on $\Lambda$ as

$$
\phi_{\lambda, p, q}^{\zeta}(\omega)= \begin{cases}\frac{1}{Z_{p, q}^{\zeta}} p^{|\omega|}(1-p)^{m-|\omega|} q^{k(\omega, \Lambda)} & \omega \in \Omega_{\Lambda}^{\zeta} \\ 0 & \omega \notin \Omega_{\Lambda}^{\zeta}\end{cases}
$$

We now say that a probability measure $\phi$ on $\Omega$ is a random-cluster measure if

$$
\phi(\omega \mid \omega(e)=\zeta(e) \forall e \notin E(\Lambda))=\phi_{\lambda, p, q}^{\zeta}(\omega)
$$

for $\phi$-a.e. $\zeta$.

\section{1 $q \geqslant 1$}

Just as for percolation, we may consider the event $E_{\infty}$ that there exists an infinite open cluster in $\omega$. By using stochastic domination one can show [5] that there exists a percolation threshold $p_{c}(q)$ such that

$$
\begin{array}{ll}
\phi_{p, q}\left(E_{\infty}\right)=0 & p<p_{c}(q) \\
\phi_{p, q}\left(E_{\infty}\right)=1 & p>p_{c}(q)
\end{array}
$$

and also that

$$
p_{c}(q) \leqslant \frac{p_{c}(1) q}{1-p_{c}(1)+p_{c}(1) q}
$$

As in the case of percolation, we may consider the random-cluster model on our family of planar graphs and obtain 
Theorem 4.1. For any rotor gadget $R$, any self-dual plane hypergraph lattice $\mathcal{H}$ and any $1 \leqslant q$,

$$
p_{c}(q)(L(R, \mathcal{H}, \rho))=p_{c}(q)\left(L\left(R, \mathcal{H}, R^{E(\mathcal{H})}\right)\right.
$$

for all $\rho \in 2^{E(\mathcal{H})}$.

Proof. We will prove the result by showing that the probability of having a set of paths joining specified terminal vertices in a copy of $R$ does not depend on the embedding of $R$. Let $A, B$, and $C$ be the terminals of $R$. Our goal is to show that

$$
\phi_{R, p, q}^{\zeta}(\omega)=\phi_{R^{\prime}, p, q}^{\zeta}(\omega)
$$

From the definition of $\phi_{R, p, q}^{\zeta}$ we see that the only things that influence the probability of a configuration $\omega$ in $R$ is the number of components $k(\omega, R)$ and the number of open edges in $R$. By the symmetry of $R$, we find that $R$ and $R^{\prime}$ have exactly the same number of configurations with a given number of open edges in $R$ and a given value of $k(\omega, R)$. Hence the probability of having a given partition of $\{A, B, C\}$ will be equal in both cases.

Thus, given two vertices $u, v \in H u b(L)$ the probability of having a path from $u$ to $v$ does not depend on the orientation of the generators used to build $L$. Likewise the probability of having an infinite path, which is equal to that of having an infinite component, does not depend on the orientations.

Since the number of vertices in a cluster is bounded by a constant times the number of vertices from the hubset it contains, we also get:

Theorem 4.2. The set of critical exponents, if they exist, for the random-cluster model on $L(R, \mathcal{H}, \rho)$ is independent of $\rho$.

\section{$4.2 q<1$}

For $q<1$ much less is known about the random-cluster model. It is not known if the model has a percolation threshold of some kind, but there are several interesting limits as $q \rightarrow 0$. One well studied example is the case where $q / p \rightarrow 0$. In this case the limiting measure is the uniform measure on the set of spanning trees of the underlying graph. For this measure Feder and Mihail [6] proved that negative association holds, as opposed to the case of the random-cluster model for $q \geqslant 1$. For general $q<1$ some type of negative correlation property is believed to hold as well but this has so far, despite considerable effort, only been proved for a small number of cases [13]. However, since our construction relies only on the equality of the conditional distributions, the random-cluster model on our lattices will display the same kind of equivalence as for $1 \leqslant q$.

\section{$5 \quad$ Further directions}

The random-cluster model is just the first of many models which show identical behaviour on all lattices in a family of the type we have constructed. The methods of [1] show that 
the behaviour of the Ising model with an external field will also be independent of $\rho$ for all members of $L(R, \mathcal{H}, \rho)$. In a similar way the results can be extended to the $q$-state Potts model with an external field. This can also be extended to e.g. bootstrap percolation. The number of walks of a given length between any pair of vertices in $\operatorname{Hub}(L)$ will be independent of $\rho$ and thereby the Green's function for a random walk on $L$ as well.

Indeed it seems hard to find any model defined in terms of nearest-neighbour interactions which will be dependent on the choice of $\rho$.

For percolation we have described how to compute $p_{c}(R)$ exactly and by Theorem 3.7 we know that the critical exponents are the same for all members of $L(R, \mathcal{H}, \rho)$. It would be interesting to extend the methods of [15] to determine their exact values as well.

Acknowledgements: The authors gratefully acknowledge the financial support and stimulating environment of the Mittag-Leffler Institute of the Swedish Royal Academy of Sciences during our visits in spring 2009. John C. Wierman thanks the Johns Hopkins University's Acheson J. Duncan Fund for the Advancement of Research in Statistics for supporting this research. We would also like to thank the referee for the constructive criticism.

\section{References}

[1] Andrén, D., and Markström, K. (2009) The Bivariate Ising polynomial of a graph. Disc. Appl. Math., to appear.

[2] Bollobás, B., and Riordan, O. (2006) Percolation. Cambridge University Press, Cambridge.

[3] Bollobás, B., and Riordan, O. (2008) Percolation on dual lattices with $k$-fold symmetry. Random Structures and Algorithms 32 463-472.

[4] Fortuin, C. M., and Kasteleyn, P. W. (1972) On the random-custer model. I. Introduction and relation to other models. Physica 57 536-564.

[5] Fortuin, C. M. (1972) On the random-custer model III. Physica 59 545-570.

[6] Feder, T. and Mihail, M. Balanced matroids. (1992) In Proceedings of the 24th Annual ACM Symposium on Theory of Computing (STOC), ACM Press, 26-38.

[7] Grimmett, G. R. (1999) Percolation (2nd ed.), Springer, New York.

[8] Grimmett, G. R. (2006) The Random-Cluster Model, Springer, Berlin.

[9] Kesten, H. (1982) Percolation Theory for Mathematicians, Birkhäuser, Boston.

[10] Scullard, C. R. (2006) Exact site percolation thresholds using a site-to-bond transformation and the star-triangle transformation. Phys. Rev. E 73016107

[11] Scullard, C. R., and Ziff, R. M. (2006) Predictions of bond percolation thresholds for the kagomé and Archimedean $\left(3,12^{2}\right)$ lattices. Phys. Rev. E 73 045102(R)

[12] Sedlock, M. R. A., and Wierman, J. C. (2009) Equality of bond percolation critical exponents for pairs of dual lattices. Physical Review E, submitted. arXiv:0903.3682. 
[13] Semple, C. and Welsh, D. J. A. (2008) Negative correlation in graphs and matroids Combinatorics, Probability and Computing 17 423-435.

[14] Sheffield, S. (2005) Random surfaces. Astérisque 304.

[15] Smirnov, S. and Werner, W. (2001) Critical exponents for two-dimensional percolation. Math. Res. Lett. 8 729-744.

[16] Tutte, W. T. (1947) A ring in graph theory. Proc. Cambridge Philos. Soc. 43 26-40.

[17] Tutte, W. T. (1998) Graph Theory as I Have Known It. Oxford Lecture Series in Mathematics and its Applications (Vol. 11), The Clarendon Press, Oxford University Press, New York.

[18] Wierman, J. C. (1992) Equality of the bond percolation critical exponents for two pairs of dual lattices. Comb. Prob. Comp. 1 95-105.

[19] Wierman, J. C. and Ziff, R. M. (2009) Self-dual planar hypergraphs and exact bond percolation thresholds. ,arXiv:0903.3135, submitted.

[20] Ziff, R. M. (2006) Generalised cell-dual-cell transformation and exact thresholds for percolation. Phys. Rev. E 73016134

[21] Ziff, R. M. and Scullard, C. R. (2006) Exact bond percolation thresholds in two dimensions. J. Phys. A: Math. Gen. 39 15083-15090 